Enferm Bras 2021;20(6):790-806

doi: $10.33233 /$ eb.v20i6.4319

\title{
REVISÃO \\ Diagnósticos de enfermagem identificados em unidade de terapia intensiva neonatal
}

Rayssa Tamyrs Almeida do Nascimento*, João Victor Batista Cabral"**, Maria Mariana Barros Melo da Silveira ${ }^{* * *}$, Amanda Tavares Xavier ${ }^{* * * *}$, Suzana de Oliveira Mangueira, D.Sc. ${ }^{\star * * \star *}$

*Graduanda do curso de Bacharelado em Enfermagem da Universidade Federal de Pernambuco, Centro Acadêmico de Vitória, Vitória de Santo Antão, PE, ${ }^{* \star}$ Enfermeiro, Especialista em Terapia Intensiva, Doutorando em Inovação Terapêutica PPGIT/UFPE, ${ }^{* * * D o c e n t e ~ d a s ~ F a c u l d a d e s ~ I n t e g r a d a s ~ d a ~ V i t o ́ r i a ~ d e ~ S a n t o ~ A n t a ̃ o, ~ V i t o r i a ~ d e ~ S a n t o ~ A n t a ̃ o, ~}$ PE, Enfermeira, Especialista em Enfermagem em Cardiologia, Doutoranda em Inovação Terapêutica PPGIT/UFPE, ${ }^{* * \star * E n f e r m e i r a, ~ E s p e c i a l i s t a ~ e m ~ E n f e r m a g e m ~ C i r u ́ r g i c a, ~}$ Doutoranda em Ciências da Saúde FCM/UPE, ${ }^{* * * * E n f e r m e i r a, ~ P r o f e s s o r a ~ A d j u n t a ~ I I, ~}$ Núcleo de Enfermagem da Universidade Federal de Pernambuco, Centro Acadêmico de Vitória, Vitória de Santo Antão, PE

Recebido em 3 de agosto de 2021; aceito em 16 de dezembro de 2021.

Correspondência: Maria Mariana Barros Melo da Silveira, Pronto Socorro Cardiológico de Pernambuco (PROCAPE/UPE), Rua dos Palmares, Santo Amaro, 50100-060 Recife PE

Rayssa Tamyrs Almeida do Nascimento: rayssawfs@hotmail.com João Victor Batista Cabral: jvbcabral@gmail.com Maria Mariana Barros Melo da Silveira: amanda-xavier@hotmail.com Amanda Tavares Xavier: marianabms@gmail.com

Suzana de Oliveira Mangueira: suzanaom@hotmail.com

\section{Resumo}

O trabalho da enfermagem exercido em uma Unidade de Terapia Intensiva Neonatal (UTIN) é um desafio constante, pois requer vigilância, habilidade, conhecimento e sensibilidade, uma vez que o paciente é bastante vulnerável e totalmente dependente da equipe que o assiste. O estudo teve como objetivo identificar os principais diagnósticos de enfermagem na UTIN presentes na literatura brasileira a partir do ano 
de 2009. Trata-se de um estudo de revisão integrativa, realizado na Biblioteca Virtual em Saúde, com os descritores UTI neonatal, diagnóstico de enfermagem e processo de enfermagem. Foram incluídos cinco artigos para análise. Os principais diagnósticos foram: Padrão ineficaz de alimentação do lactente; Integridade da pele prejudicada; Risco de infecção; Padrão respiratório ineficaz; Troca de gases prejudicada e Desobstrução ineficaz das vias aéreas. A identificação correta de tais diagnósticos pode subsidiar uma assistência de enfermagem mais efetiva.

Palavras-chave: unidade de terapia intensiva neonatal; diagnóstico de enfermagem; processo de enfermagem.

\section{Abstract}

\section{Nursing diagnoses identified in a neonatal intensive care unit}

The nursing work performed in a Neonatal Intensive Care Unit (NICU) is a constant challenge, as it requires vigilance, skill, knowledge and sensitivy, since the patient is quite vulnerable and totally dependent on the team that assists him. The study aimed to identify the main nursing diagnoses in the NICU present in the Brazilian literature as of 2009. This is an integrative review study, carried out in the Virtual Health Library, with the descriptors neonatal ICU, nursing diagnosis and nursing process. Five papers were included for analysis. The main diagnoses were: Ineffective infant feeding pattern; Impaired skin integrity; Risk of infection; Ineffective respiratory pattern; Impaired gas exchange and ineffective airway clearance. The correct identification of such diagnoses can support more effective nursing care.

Keywords: intensive care units, neonatal; nursing diagnosis; nursing process.

\section{Resumen}

\section{Diagnósticos de enfermería identificados en unidad de cuidado intensivo neonatal}

El trabajo de enfermería que se realiza en una Unidad de Cuidados Intensivos Neonatal (UCIN) es un desafío constante, pues requiere vigilancia, habilidad, conocimiento y sensibilidad, ya que el paciente es bastante vulnerable y totalmente dependiente del equipo que lo atiende. El estudio tuvo como objetivo identificar los principales diagnósticos de enfermería en la UCIN presentes en la literatura brasileña desde 2009 en adelante. Se trata de un estudio de revisión integradora, realizado en la Biblioteca Virtual en Salud, con los descriptores UCI neonatal, diagnóstico de enfermería y proceso de enfermería. Se incluyeron cinco artículos para el análisis. Los principales diagnósticos fueron: patrón de alimentación infantil ineficaz; Integridad de la piel deteriorada; Riesgo de infección; Patrón de respiración ineficaz; Intercambio de gases 
alterado y Desobstrucción ineficaz de las vías respiratorias. La identificación correcta de dichos diagnósticos puede contribuir a una atención de enfermería más eficaz.

Palabras-clave: unidades de cuidado intensivo neonatal; diagnóstico de enfermería; proceso de enfermería.

\section{Introdução}

A enfermagem tem como foco de trabalho o cuidado ao ser humano em todas as fases do desenvolvimento e vem ampliando constantemente seu conhecimento. $O$ trabalho da enfermagem exercido em uma Unidade de Terapia Intensiva Neonatal (UTIN) é um desafio constante, pois requer vigilância, habilidade, conhecimento e sensibilidade, uma vez que o paciente é bastante vulnerável e totalmente dependente da equipe que o assiste [1]. As UTIN foram criadas com o objetivo de atender recém-nascidos (RN) mediante a realização de procedimentos complexos e invasivos aliados à utilização de tecnologias cada vez mais específicas [2].

Atualmente, com o avanço tecnológico, cada vez mais os neonatos de alto risco, em especial os prematuros e/ou de baixo peso ao nascer, são beneficiados e possuem maiores chances de sobrevida. Destaca-se que a prematuridade predispõe o RN a inúmeras complicações $[3,4]$. Entretanto, a redução da mortalidade neonatal, advinda do avanço tecnológico, acentua a morbidade nessa população específica, em decorrência do elevado tempo de internação que se faz necessário para garantia de sobrevida destes pacientes [4].

O processo de trabalho de enfermagem em UTIN é caracterizado por atividades assistenciais complexas que exigem alta competência técnica e científica, afinal, a tomada de decisões imediatas e adoção de condutas seguras estão diretamente relacionadas à vida e morte dos pacientes. Nesse contexto, é importante prover e manter o pessoal de enfermagem qualificado e adequadamente dimensionado para desenvolver a assistência de enfermagem com qualidade e segurança [2].

A utilização do Processo de Enfermagem (PE) como referencial da Sistematização da Assistência de Enfermagem (SAE) na UTIN é fundamental, pois a assistência qualificada não deve se limitar a garantir a sobrevida do prematuro, mas também planejar ações e implementá-las conforme as necessidades do cuidado [5,4].

A SAE configura-se como uma metodologia para organizar e sistematizar o cuidado, com base nos princípios do método científico. Tem como objetivos identificar as situações de saúde-doença e as necessidades de cuidados de enfermagem, bem como subsidiar as intervenções de promoção, prevenção, recuperação e reabilitação da saúde do indivíduo, família e comunidade [2,6]. 
O cuidado sistematizado busca promover uma assistência holística, individualizada e mais qualificada ao paciente, consolidando o comprometimento e a responsabilidade do profissional perante os pacientes, familiares e comunidade. Nesse sentido, proporciona ações tanto na área assistencial quanto administrativa, o que facilita a organização do trabalho e dos serviços da equipe de enfermagem [2].

O PE é considerado a base de sustentação da SAE [2], definido como um método por meio do qual a estrutura teórica da enfermagem é aplicada à prática. É estruturado em cinco fases: investigação, diagnóstico, planejamento, implementação e avaliação [2,4-8].

A segunda fase do PE compõe-se dos Diagnósticos de Enfermagem (DE) que, segundo a NANDA Internacional (NANDA-I), trata-se de "um julgamento clínico sobre a resposta de um indivíduo, uma família ou uma comunidade com relação a problemas de saúde reais ou potenciais/processos de vida que fornecem a base para uma terapia definitiva, que busca alcançar resultados nos quais a enfermagem é necessária" $[2,6,7]$. Cada diagnóstico apresenta componentes estruturais: o título, a definição, os fatores relacionados ou fatores de risco e as características definidoras [2].

Diante desse contexto em que a SAE e o PE se configuram como ferramentas fundamentais para a assistência em UTIN e em virtude da escassez de estudos que identifiquem os DE no ambiente frequentes na UTIN, faz-se necessário compilar e descrever a ocorrência dos DE presentes na literatura que subsidiem o cuidado de enfermagem específico para RN crítico. Este estudo tem como objetivo identificar os principais diagnósticos de enfermagem na UTIN presentes na literatura brasileira a partir do ano de 2009, ano da promulgação da Resolução do Conselho Federal de Enfermagem (COFEN) 358/2009, que estabelece a implantação da SAE e PE em todos os ambientes em que ocorra assistência de enfermagem.

\section{Métodos}

Este estudo fundamentou-se no método de revisão integrativa de literatura com a finalidade de alcançar o objetivo proposto. Foi realizado por meio de seis etapas distintas: $1^{\text {a }}$ etapa: Identificação do tema e seleção da hipótese ou questão de pesquisa para a elaboração da revisão integrativa; $2^{\underline{a}}$ etapa: Estabelecimentos dos critérios de inclusão e exclusão de estudo/amostragem ou busca da literatura; $3^{\underline{a}}$ etapa: Definição das informações a serem extraídas de estudos selecionados; 4aㅡ etapa: Avaliação dos estudos incluídos na revisão integrativa; $5^{\underline{a}}$ etapa: interpretação dos resultados; $6^{\underline{a}}$ etapa: Apresentação da revisão/síntese do conhecimento [9]. 
1' Etapa: O tema deste estudo é a aplicação do PE, especificamente a etapa dos Diagnósticos de Enfermagem (DE), no ambiente de UTI Neonatal. O estudo foi conduzido pela seguinte questão norteadora: Quais os principais DE identificados na UTI Neonatal presentes nas publicações brasileiras dos últimos 10 anos?

2ª Etapa: A partir da estratégia do acrônimo PICOS [10], foram considerados elegíveis como critérios de inclusão artigos cujos objetivos fossem a aplicação do PE no ambiente de UTI Neonatal, publicados em língua portuguesa, com texto completo, disponíveis de forma gratuita eletronicamente, indexados na Biblioteca Virtual em Saúde (BVS), nas bases Literatura Latinoamericana y del Caribe em Ciências de La Salud (Lilacs), Índice Bibliográfico Espanhol em Ciências da Saúde (IBECS), Medical Literature Analysis and Retrieval System Online (Medline) e Base de Dados da Enfermagem (BDENF), no período 2009 a 2018, e que contivessem os descritores utilizados na busca nos seus título, resumo ou assunto. Excluíram-se editoriais e artigos de revisão.

A amostragem se deu a partir da coleta de dados secundários por meio de levantamento e análise bibliográfica de publicações resultantes da busca dos artigos pelos descritores selecionados no DeCS (Descritores em Ciência da Saúde / http://desc.bvs.br): UTI Neonatal; Diagnóstico de Enfermagem; e Processo de Enfermagem, com cruzamento realizado com o operador booleano "and", conforme descrito no quadro 1.

Quadro 1 - Cruzamento de descritores na BVS com utilização do operador booleano "AND"

\begin{tabular}{|l|l|l|l|}
\hline Cruzamento & $\begin{array}{l}\text { Artigos } \\
\text { Encontrados }\end{array}$ & Artigos excluídos & Artigos incluídos \\
\hline UTI Neonatal and DE and PE & 1 & 0 & 1 \\
\hline UTI Neonatal and DE & 54 & 46 & 8 \\
\hline UTI Neonatal and PE & 183 & 182 & 1 \\
\hline Total & 238 & 228 & 10 \\
\hline
\end{tabular}

3ㄹ. Etapa: As informações extraídas dos artigos foram de caráter descritivo, diretamente relacionadas ao objetivo deste estudo que é identificar e descrever os principais DE na UTI Neonatal presentes na literatura. A avaliação crítica da qualidade dos dados dos estudos foi realizada pelo método Oxford Centre Evidence Based Medicine [11].

4를 Etapa: A avaliação dos estudos baseou-se nos critérios de inclusão e exclusão, sendo realizada, primariamente, a leitura dos títulos e resumos, por cada autor separadamente, o que resultou no achado de 10 artigos. Secundariamente, procedeu-se sua leitura na íntegra por cada autor, sendo assim definidos os estudos incluídos como amostra. Dos 
10 estudos, 3 foram excluídos por não serem realizados no ambiente de UTI Neonatal e 02 por não descrever nenhum DE, sendo a amostra deste estudo composta por 05 artigos.

5ª Etapa: A interpretação dos resultados consistiu na extração dos dados relacionados às características bibliométricas dos estudos, a descrição das etapas do PE e dos DE presentes nos estudos, sendo estes padronizados ou não na NANDA I.

6 Etapa: A apresentação da revisão foi realizada por meio de quadros descritivos e tabela contendo: numeração do artigo, título, base, autores, local de realização do estudo, tipo de estudo e objetivo (Quadro 2); numeração do artigo, etapas do PE e DE (Quadro 3); e numeração do artigo, total de DE e Padronização do DE pela NANDA I (Quadro 4); e DE com número do artigo, descrição de valor absoluto e percentual (Tabela I).

\section{Resultados}

De acordo com o Quadro 2, entre os cinco artigos selecionados, dois estavam indexados na base de dados BDENF e LILACS e um na Medline. Todos os estudos foram realizados no cenário de UTI Neonatal e como tipo de estudo, três adotaram a abordagem descritiva retrospectiva quantitativa, um transversal quantitativo e um do tipo intervenção. Todos os estudos tiveram como objetivo descrever o PE, apresentando diretamente os DE na UTIN. 
Quadro 2 - Apresentação da revisão de acordo com: numeração do artigo, título, base, autores, local de realização do estudo, tipo de estudo e objetivo.

\begin{tabular}{|c|c|c|c|c|c|c|}
\hline $\mathrm{N}^{0}$ & Título & Base & Autores & Local & $\begin{array}{l}\text { Tipo de } \\
\text { estudo }\end{array}$ & Objetivo \\
\hline 1 & $\begin{array}{l}\text { Instrumentos para o } \\
\text { processo de } \\
\text { enfermagem do } \\
\text { neonato prétermo à luz } \\
\text { da teoria de Dorothy } \\
\text { Johnson }\end{array}$ & BDENF & $\begin{array}{l}\text { Fialho FA, } \\
\text { et al. }\end{array}$ & $\begin{array}{l}\text { UTl } \\
\text { Neonatal }\end{array}$ & Intervençäo & $\begin{array}{l}\text { Apresentar } \\
\text { três } \\
\text { instrumentos } \\
\text { elaborados } \\
\text { sob a luz da } \\
\text { Teoria de } \\
\text { Enfermagem } \\
\text { do Modelo do } \\
\text { Sistema } \\
\text { Comportament } \\
\text { al de Dorothy } \\
\text { Johnson para } \\
\text { subsidiar o PE } \\
\text { em uma UTI } \\
\text { Neonatal }\end{array}$ \\
\hline 2 & $\begin{array}{l}\text { Diagnósticos de } \\
\text { enfermagem de } \\
\text { prematuro sob } \\
\text { cuidados intermediários }\end{array}$ & MEDLINE & $\begin{array}{l}\text { Del'Angelo } \\
\mathrm{N} \text {, et al. }\end{array}$ & $\begin{array}{l}\text { UTI } \\
\text { Neonatal }\end{array}$ & $\begin{array}{l}\text { Descritivo } \\
\text { Retrospectivo } \\
\text { Quantitativo }\end{array}$ & $\begin{array}{l}\text { Identificar DE } \\
\text { em } \\
\text { prematuros } \\
\text { assistidos em } \\
\text { uma UTI } \\
\text { Neonatal }\end{array}$ \\
\hline 3 & $\begin{array}{l}\text { Diagnósticos de } \\
\text { enfermagem de recém- } \\
\text { nascidos com sepse } \\
\text { em uma unidade de } \\
\text { terapia intensiva } \\
\text { neonatal }\end{array}$ & LILACS & $\begin{array}{l}\text { Santos } \\
\text { APS, et al. }\end{array}$ & $\begin{array}{l}\text { UTI } \\
\text { Neonatal }\end{array}$ & $\begin{array}{l}\text { Transversal } \\
\text { Quantitativo }\end{array}$ & $\begin{array}{l}\text { Elaborar os } \\
\text { DE em recém- } \\
\text { nascidos com } \\
\text { sepse em uma } \\
\text { UTI Neonatal }\end{array}$ \\
\hline 4 & $\begin{array}{l}\text { Identificação do perfil e } \\
\text { diagnósticos de } \\
\text { enfermagem do } \\
\text { neonato com } \\
\text { cardiopatia congênita }\end{array}$ & BDENF & $\begin{array}{l}\text { Urakawa } \\
\text { IT, } \\
\text { Kobayashi } \\
\text { RIM }\end{array}$ & $\begin{array}{l}\text { UTI } \\
\text { Neonatal }\end{array}$ & $\begin{array}{l}\text { Descritivo } \\
\text { Retrospectivo } \\
\text { Quantitativo }\end{array}$ & $\begin{array}{l}\text { Caracterizar o } \\
\text { perfil e } \\
\text { identificar os } \\
\text { DE do } \\
\text { Neonato com } \\
\text { Cardiopatia } \\
\text { Congênita }\end{array}$ \\
\hline 5 & $\begin{array}{l}\text { Diagnósticos de } \\
\text { enfermagem em } \\
\text { recém-nascido com } \\
\text { alteraçôes glicêmicas }\end{array}$ & LILACS & $\begin{array}{l}\text { Oliveira } \\
\text { SIM, } \\
\text { Souza NL, } \\
\text { Costa e } \\
\text { Silva RK }\end{array}$ & $\begin{array}{l}\text { UTI } \\
\text { Neonatal }\end{array}$ & $\begin{array}{l}\text { Descritivo } \\
\text { Retrospectivo } \\
\text { Quantitativo }\end{array}$ & $\begin{array}{l}\text { Identificar os } \\
\text { DE mais } \\
\text { frequentes } \\
\text { entre recém- } \\
\text { nascidos com } \\
\text { alterações } \\
\text { glicêmicas }\end{array}$ \\
\hline
\end{tabular}

Conforme o Quadro 3, entre os cinco artigos selecionados, três adotaram as cinco etapas do PE para execução de seu estudo e dois executaram apenas as etapas de coleta de dados e DE. Os artigos 1 e 2 apresentaram o maior número de DE, sendo 26 e 21, respectivamente, seguidos do artigo 3 com 13, artigo 4 com 12 e o artigo 5 com $9 \mathrm{DE}$. 
Quadro 3 - Apresentação da revisão de acordo com: numeração do artigo, etapas do $P E$ e $D E$

\begin{tabular}{|c|c|c|}
\hline $\mathrm{N}^{0}$ & Etapas do PE & $\mathrm{DE}$ \\
\hline 1 & $\begin{array}{l}\text { - Coleta de Dados; } \\
\text { - Diagnósticos de Enfermagem; } \\
\text { - Planejamento de Enfermagem; } \\
\text { - Intervenções de Enfermagem; } \\
\text { - Avaliação de Enfermagem. }\end{array}$ & 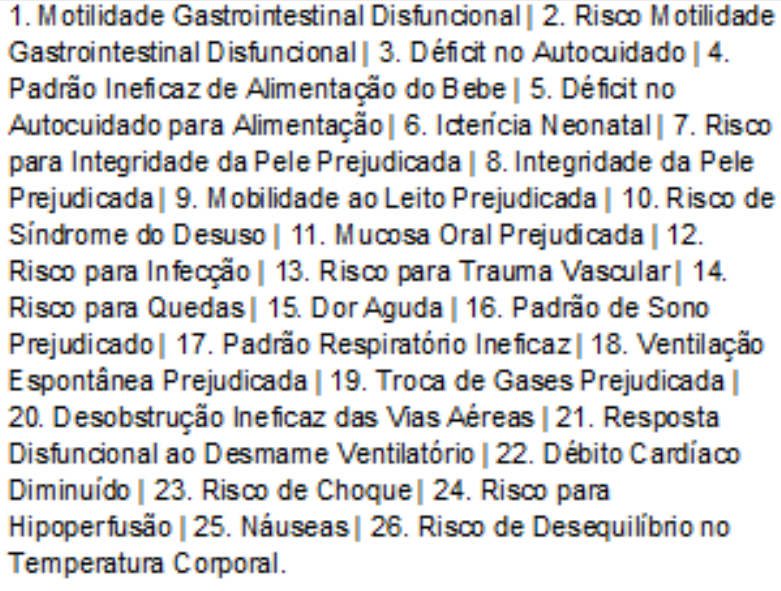 \\
\hline 2 & $\begin{array}{l}\text { - Coleta de Dados; } \\
\text { - Diagnósticos de Enfermagem; } \\
\text { - Planejamento de Enfermagem; } \\
\text { - Intervenções de Enfermagem; } \\
\text { - Avaliação de Enfermagem. }\end{array}$ & $\begin{array}{l}\text { 1. Privação do Sono | 2. Risco de Infecção | 3. Processos } \\
\text { Familiares Interrompidos | 4. Dor| 5. Risco de Integridade da } \\
\text { Pele Prejudicada | 6. Atraso no Crescimento e no } \\
\text { Desenvolvimento |7. Risco de Aspiração | 8. Amamentação } \\
\text { Interrompida | 9. Termorregulação Ineficaz|10. Mucosa Oral } \\
\text { Prejudicada | 11. Risco de Vínculo Pais/filho Alterado | 12. } \\
\text { Risco de Desequilibrio na Temperatura Corporal |13. Padrão } \\
\text { Respiratório Ineficaz| 14. Padrão Ineficaz de Alimentação do } \\
\text { Bebê| 15. Ventilação Espontânea Prejudicada | 16. Proteção } \\
\text { Ineficaz | 17. Capacidade Adaptativa Diminuida Intracraniana | } \\
\text { 18. Integridade da Pele Prejudicada | 19. Desobstrução } \\
\text { Ineficaz de Vias Aéreas | 20. Constipação | 21. Nutrição } \\
\text { Desequilibrada. }\end{array}$ \\
\hline 3 & $\begin{array}{l}\text { - Coleta de Dados; } \\
\text { - Diagnósticos de Enfermagem. }\end{array}$ & $\begin{array}{l}\text { 1. Risco de Choque | 2. Risco de Desequilíbrio do Volume de } \\
\text { Líquidos | 3. Motilidade Gastrintestinal Disfuncional| 4. } \\
\text { Icterícia Neonatal| 5. Troca de Gases Prejudicada | 6. Padrão } \\
\text { Respiratório Ineficaz| 7. Risco de Sangramento | 8. Risco de } \\
\text { Perfusão Renal Ineficaz | 9. Risco de Atraso no } \\
\text { Desenvolvimento | 10. Débito Cardíaco Diminuído | } 11 . \\
\text { Hipotermia | 12. Risco de Vínculo Prejudicado | 13. } \\
\text { Desobstrução Ineicaz de Vias Aéreas. }\end{array}$ \\
\hline 4 & $\begin{array}{l}\text { - Coleta de Dados; } \\
\text {-Diagnósticos de Enfermagem. }\end{array}$ & $\begin{array}{l}\text { 1. Risco para Infecção | 2. Integridade da Pele Prejudicada|3. } \\
\text { Risco para Alteraçấo da Temperatura | 4. Risco para Alteração } \\
\text { no Volume de Liquidos | 5. Risco para Alteração na FC, PAe } \\
\text { Ritmo Cardíaco | 6. Risco para Alteração no Padräo } \\
\text { Respiratório | 7.Limpeza Ineficaz de Vias Aéreas Superiores | } \\
\text { 8.Risco para Prejuizo na Integridade da Pele | 9.Risco para } \\
\text { Inapetência | 10. Excesso de Volume de Liquidos|11. Risco } \\
\text { para Aspiração | 12. Risco para Alteração no Metabolismo de } \\
\text { Glicose. }\end{array}$ \\
\hline 5 & $\begin{array}{l}\text {-Coleta de Dados; } \\
\text {-Diagnósticos de Enfermagem. }\end{array}$ & $\begin{array}{l}\text { 1. Nutrição Desequilibrada Menos que as Necessidades } \\
\text { Corporais | 2. Risco de Glicemia Instável| } 3 \text {. Padrão Ineficaz } \\
\text { de Alimentação do Bebê| 4. Risco de Crescimento } \\
\text { Desproporcional | 5. Patemidade ou Maternidade Prejudicada | } \\
\text { 6. Amamentação Ineficaz| 7. Troca de Gases Prejudicada | } 8 \text {. } \\
\text { Amamentação Interrompida | 9. Risco de infecção. }\end{array}$ \\
\hline
\end{tabular}

Quadro 4 - Apresentação da revisão de acordo com: numeração do artigo e total de DE e padronização dos DE na NANDA (ver PDF anexo) 
Quanto à padronização do DE de acordo com a NANDA I, verificou-se que 100\% dos DE apresentados no artigo 5 estavam padronizados, seguidos dos artigos 3 e 1 com $92,30 \%$ de padronização e o artigo 2 apresentou 80,95\% de padronização. $O$ artigo 4 apresentou alto percentual de DE não padronizados e, os aqueles que foram considerados padronizados apresentavam erros na escrita das preposições.

Conforme pode ser visto na Tabela 1, os DE com maior ocorrência entre as cinco publicações foram: Padrão ineficaz de alimentação do lactente; Integridade da pele prejudicada; Risco de infecção; Padrão respiratório ineficaz; Troca de gases prejudicada; e Desobstrução ineficaz das vias aéreas, presentes em três artigos, cada um perfazendo $5 \%$ do total de DE encontrados nos estudos. 
Tabela I - Apresentação da revisão de acordo com: DE e número do artigo, descrição de valor absoluto e percentual

\begin{tabular}{|c|c|c|}
\hline $\mathrm{DE}^{\left(\mathrm{N}^{2} \text { do Artilos) }\right.}$ & $\mathbf{N}$ & $\%$ \\
\hline Padrão ineicaz de alimentação do bebê 1.205 & 3 & 5,0 \\
\hline Integridade da pele prejudicada 1.204 & 3 & 5,0 \\
\hline Risco de in feccão ${ }^{1,205}$ & 3 & 5,0 \\
\hline Padrão respiratório ine fica $z^{1,203}$ & 3 & 5,0 \\
\hline Troca de gases prejudicada 1,3 e 5 & 3 & 5,0 \\
\hline Desobstrução ine ficaz das vias aéreas 1,203 & 3 & 5,0 \\
\hline Amamentação interrompida $2 \circ 5$ & 2 & 3,3 \\
\hline Ris $\infty$ de integridade da pele prejudicada ${ }^{102}$ & 2 & 3,3 \\
\hline Motilidade gastrointestinal disfuncional ${ }^{103}$ & 2 & 3,3 \\
\hline Icterícia neonatal $^{1 \text { e } 3}$ & 2 & 3,3 \\
\hline Mucosa oral prejudicada ${ }^{1 e 2}$ & 2 & 3,3 \\
\hline Ventilação espontânea prejudicada ${ }^{12}$ & 2 & 3,3 \\
\hline Débito cardíaco diminuido 103 & 2 & 3,3 \\
\hline Risco de choque ${ }^{103}$ & 2 & 3,3 \\
\hline Ris $\infty$ de desequilibrio na temperatura corporal ${ }^{102}$ & 2 & 3,3 \\
\hline Déficit no autocuidado para alimentação ${ }^{1}$ & 1 & 1,7 \\
\hline Mobilidade ao leito prejudicada 1 & 1 & 1,7 \\
\hline Risco de motilidade gastrointestinal disfuncional ${ }^{1}$ & 1 & 1,7 \\
\hline Déficit no autocuidado ${ }^{1}$ & 1 & 1,7 \\
\hline Risco de sindrome de desuso ${ }^{1}$ & 1 & 1,7 \\
\hline Risco para trauma vascular ${ }^{1}$ & 1 & 1,7 \\
\hline Risco para queda 1 & 1 & 1,7 \\
\hline Dor aguda ${ }^{1}$ & 1 & 1,7 \\
\hline Padrão de sono prejudicado ${ }^{1}$ & 1 & 1,7 \\
\hline Resposta disfuncional ao derrame ventilatório ${ }^{4}$ & 1 & 1,7 \\
\hline Risco para hipoperfusão ${ }^{1}$ & 1 & 1,7 \\
\hline Náuseas ${ }^{1}$ & 1 & 1,7 \\
\hline Privaçäo de sono ${ }^{2}$ & 1 & 1,7 \\
\hline Processo familiares interrompidos 2 & 1 & 1,7 \\
\hline Dor ${ }^{2}$ & 1 & 1,7 \\
\hline Atraso no desenvolvimento e no crescimento 2 & 1 & 1,7 \\
\hline Risco de aspiração 2 & 1 & 1,7 \\
\hline Termorregulação ine ficaz 2 & 1 & 1,7 \\
\hline Risco de vínculo pais/filho alterados ${ }^{2}$ & 1 & 1,7 \\
\hline Proteção ineficaz ${ }^{2}$ & 1 & 1,7 \\
\hline Capacidade adaptativa diminuida intracraniana 2 & 1 & 1,7 \\
\hline Constipação $=$ & 1 & 1,7 \\
\hline Nutriçẩo desequilibrada ${ }^{2}$ & 1 & 1,7 \\
\hline Risco de desequilíbrio do volume de liquido ${ }^{3}$ & 1 & 1,7 \\
\hline Ris $c 0$ de sangramento 3 & 1 & 1,7 \\
\hline Risco de perfusão renal ine ficaz ${ }^{3}$ & 1 & 1,7 \\
\hline Risco de atraso no desenvolvimento ${ }^{3}$ & 1 & 1,7 \\
\hline Hipotermia ${ }^{3}$ & 1 & 1,7 \\
\hline Risco de vinculo prejudicado ${ }^{3}$ & 1 & 1,7 \\
\hline Risco para aspiração ${ }^{4}$ & 1 & 1,7 \\
\hline Excesso de volume de líquido ${ }^{4}$ & 1 & 1,7 \\
\hline Risco para in fecção ${ }^{4}$ & 1 & 1,7 \\
\hline Risco para alteração da temperatura ${ }^{4}$ & 1 & 1,7 \\
\hline Risco para alteração no volume de liquido ${ }^{4}$ & 1 & 1,7 \\
\hline Risco para alteração na $\mathrm{FC}, \mathrm{PA}$ e ritmo cardiaco $(\mathrm{RC})^{4}$ & 1 & 1,7 \\
\hline Risco para alteração no padrão respiratório ${ }^{4}$ & 1 & 1,7 \\
\hline Limpeza ineficaz de vias aéreas superiores ${ }^{4}$ & 1 & 1,7 \\
\hline Risco para prejuizo na integridade da pele ${ }^{4}$ & 1 & 1,7 \\
\hline Risco para inapetência 4 & 1 & 1,7 \\
\hline Risco para alterações no metabolismo de glicose $e^{4}$ & 1 & 1,7 \\
\hline Nutrição desequilibrada: a menos que as necessidades corporais ${ }^{5}$ & 1 & 1,7 \\
\hline Ris $\infty$ de glicemia instável ${ }^{5}$ & 1 & 1,7 \\
\hline Risco de patemidade ou maternidade prejudicada ${ }^{5}$ & 1 & 1,7 \\
\hline Amamentação ineicaz ${ }^{5}$ & 1 & 1,7 \\
\hline
\end{tabular}

\section{Discussão}

A análise das publicações permitiu fazer uma relação da padronização dos diagnósticos com os DE com maior ocorrência, que foram: Padrão ineficaz de alimentação do lactente; Integridade da pele prejudicada; Risco de infecção; Padrão 
respiratório ineficaz; Troca de gases prejudicada e Desobstrução ineficaz das vias aéreas.

As limitações fisiológicas de muitos prematuros, acrescidos dos frequentes problemas respiratórios apresentados, da necessidade de nutrição parenteral, gástrica ou entérica, dentre outros aspectos, justificam a identificação dos DE "Padrão Ineficaz de Alimentação do Lactente" e "Nutrição Desequilibrada: menos do que as necessidades corporais". Neste contexto, deve-se refletir sobre a sondagem gástrica enquanto um procedimento imprescindível no auxílio à nutrição de prematuros, com até 32 semanas de idade gestacional, aproximadamente, pois os mesmos ainda não conseguem coordenar os reflexos de sucção e de deglutição $[6,12]$.

O DE "Integridade da Pele Prejudicada" considera as lesões cutâneas, na epiderme e derme, que ocasionam falhas na primeira linha de defesa do organismo. Alguns autores observaram em seus estudos sobre DE em UTI, a ocorrência da Integridade da pele prejudicada em $10 \%$ e $88,3 \%$ dos pacientes internados. A Integridade da pele prejudicada caracterizou-se principalmente pelo rompimento da superfície da pele, seja em decorrência de um trauma ou pelo desenvolvimento de lesões por pressão, devido à imobilidade. Fatores de desenvolvimento e mecânicos, além de incisões cirúrgicas, estiveram relacionados a esse DE [2].

A presença do DE "Risco de Integridade da Pele Prejudicada" por vezes é subestimada nos registros dos enfermeiros, pois os prematuros apresentam diversos fatores relacionados como extremo de idade, além da presença de fatores mecânicos como adesivos para fixação de cateteres e sondas, uso de fraldas e as proeminências ósseas $[12,16]$. Cabe assinalar que a pele do recém-nascido pré-termo é delicada e propensa a lesões, sendo fundamental que a equipe de enfermagem esteja sempre atenta aos cuidados diários com o objetivo de minimizar comprometimento da integridade da pele [12]. Outro estudo identificou a presença de integridade da pele prejudicada estando presentes em 8,7\% dos RN [13].

O DE "Risco de Infecção" é definido como o risco aumentado de ser invadido por organismos patogênicos. Para esse DE, os principais fatores de risco apresentados na população em estudo foram: procedimentos invasivos, alterações laboratoriais, incisão cirúrgica, dissecção venosa e úlcera por pressão. O "Risco de Infecção" pode ser influenciado pelo número de profissionais que lida diretamente com o paciente, a quantidade e o tipo de procedimentos invasivos, a forma de assistência que é dispensada e o tempo de hospitalização. Esse diagnóstico descreve um estado que pode comprometer a recuperação de pacientes internados em uma UTI, por isso é importante que o enfermeiro realize o DE e elabore um plano de cuidados, pois, assim, 
sua equipe estará prestando uma assistência de enfermagem com medidas eficazes no controle de infecção [2].

Embora o DE "Risco de Infecção" tenha sido frequente entre os prematuros, considera-se que a quase totalidade deles deveria apresentar esse DE, pois além de consonante com a ideia de que todos os neonatos estão sujeitos à infecção devido à transição do meio estéril (útero materno) para ambiente contaminado, há ainda a fragilidade biológica do prematuro com imaturidade de diversos sistemas, em especial o imunológico. Além disso, destaca-se a realização de procedimentos de alta complexidade de apoio ao diagnóstico e à terapêutica e o longo período de internação, tornando, portanto, os RN mais suscetíveis a infecções e ao adoecimento [13].

O estudo identificou, ainda, a presença de diagnósticos relacionados ao comprometimento respiratório dos neonatos. O diagnóstico "Padrão respiratório ineficaz" tem como definição: inspiração e/ou expiração que não proporciona ventilação adequada $[16,17]$. Já o diagnóstico "Troca de gases prejudicada" refere-se à alteração na oxigenação ou eliminação do dióxido de carbono através da membrana alvéolocapilar [16,18]. A monitoração do desempenho respiratório é fundamental, principalmente, em pacientes com Insuficiência Cardíaca Congestiva (ICC). O edema pulmonar e a hipoxemia, secundários ao desequilíbrio da relação ventilação-perfusão ocasionado pela doença, acarretam prejuízos tanto na oxigenação quanto na eliminação do dióxido de carbono através da membrana alvéolo-capilar. Desta forma, a avaliação de indicadores clínicos da troca gasosa de pacientes com ICC é pertinente e pode contribuir para a identificação mais apropriada de diagnósticos de enfermagem nesta população [19]. Este diagnóstico guarda forte relação com o bom funcionamento cardíaco e respiratório.

O DE "Desobstrução ineficaz de vias aéreas" é definido como a incapacidade de eliminar secreções ou obstruções do trato respiratório para manter uma via aérea desobstruída [16]. Se a desobstrução ineficaz de vias aéreas não for adequadamente tratada, poderá acarretar outras alterações como, por exemplo, padrão respiratório ineficaz ou troca de gases prejudicada, o que resultaria em sofrimento respiratório e até morte [2]. Dessa forma, é fundamental que a equipe de enfermagem atente para que a função respiratória do neonato seja monitorada constantemente e as suas vias aéreas mantidas pérvias.

\section{Conclusão}

O estudo identificou que os DE mais frequentes nos neonatos internados em UTI estão relacionados às necessidades de nutrição, proteção e respiração alteradas. A 
identificação dos DE permite ao enfermeiro subsídios para prestar uma assistência de qualidade, com base nos princípios científicos, buscando identificar a situação saúdedoença reais do $\mathrm{RN}$, tendo como referência a NANDA I. Os cuidados necessários devem ser prestados de forma holística, humanizada e sistematizada, possibilitando alcançar os resultados esperados pelo profissional de enfermagem.

Recomenda-se a realização de estudos de validação dos principais diagnósticos encontrados como mais frequentes nos neonatos, de forma a clarificar tais fenômenos e direcionar a identificação destes diagnósticos de forma mais acurada na prática clínica e que possa, assim, proporcionar subsídios para uma assistência de enfermagem mais científica e de qualidade.

\section{Conflito de interesse}

Os autores declaram não ter conflito de interesse.

\section{Fonte de financiamento}

Sem fonte de financiamento.

\section{Contribuição dos autores}

Redação: Nascimento RTA, Cabral JVB; Estruturação do texto: Nascimento RTA, Mangueira SO; Coleta de dados: Nascimento RTA; Discussão: Nascimento RTA; Análise dos dados: Cabral JVB, Silveira MMM, Xavier AT; Revisão do texto: Cabral JVB, Silveira MMM, Xavier AT, Mangueira SO.

\section{Referências}

1. Gomes AG, Hahn GV. Manipulação do recém-nascidos internados em UTI: alerta a enfermagem. Revista Destaques Acadêmicos CCBS/UNIVATES [Internet]. 2011 [cited 2021 Dec 16];a3,N.3, CCBS/UNIVATES. Available from:

https://portaldeboaspraticas.iff.fiocruz.br/biblioteca/manipulac\%CC\%A7a\%CC\%83o-dorecem-nascido-internado-em-uti-alerta-a-enfermagem/

2. Guedes DMB, Rossato LM, Oliveira EA. Diagnósticos de enfermagem mas frequentes em uma unidade de terapia intensiva pediátrica. Rev Enferm UFSM 2015;5(3):476-485. Available from: https://pesquisa.bvsalud.org/portal/resource/pt/biblio-1034337

3. Lopes LC, Paes IADC. Possíveis diagnósticos e intervenções da enfermagem a neonatos em fototerapia. Revista Científica da FHO|UNIARARAS [Internet]. 2015 [cited 2021 Dec 16];3(2). Available from: http://www.uniararas.br/revistacientifica/_documentos/art.007-2016.pdf

4. Castro ACO, Duarte DD, Diniz IA. Intervenção do enfermeiro as crianças atendidas no ambulatório de seguimento do recém-nascido de risco. Revista de Enfermagem do Centro-Oeste Mineiro 2017;7:e1159. doi: 10.19175/recom.v7i0.1159

5. Moreira RAN, Pereira LDB, Silveira AEOB, Barros LM, Frota NM, Luna IT. Sistematização da assistência de enfermagem em unidade neonatal. Cogitare Enferm 2012;17(4):710-6. doi: 10.5380/ce.v17i4.30379 
6. Moraes-Filho IM, Souza GB, Nascimento FNN, Santos JLA, Carvalho MR. Checklist do recém-nascido: principais diagnósticos de enfermagem mediante intercorrências e susceptibilidade das mesmas no neonatal. Rev Cient Sena Aires [Internet]. 2017 [cited 2021 Dec 16];6(1):30-48. Available from:

http://revistafacesa.senaaires.com.br/index.php/revisa/article/view/276/178

7. Melo LL, Santos MS, Duran ECM. Unidade de terapia intensiva pediátrica: diagnósticos e intervenções de enfermagem mais frequentes. Rev Enferm UFPE on line 2014;(supl. 1):2342-9. doi: 10.5205/reuol.5927-50900-1-SM.0807supl201420

8. Moura RCA, Cosson ICO. Implantação da sistematização da assistência de enfermagem em uma unidade neonatal na Amazônia ocidental. Cuidarte Enfermagem [Internet]. 2013 [cited 2021 Dec 16];7(1):33-7. Available from: https://pesquisa.bvsalud.org/portal/resource/pt/biblio-1027870

9. Mendes KDS, Silveira RCCP, Galvão CM. Revisão integrativa: método de pesquisa para a incorporação de evidencias na saúde e na enfermagem. Texto Contexto Enferm 2008;17(4):758-64. doi: 10.1590/S0104-07072008000400018

10. Galvão TF, Pereira MG. Revisões sistemáticas da literatura: passos para sua elaboração. Epidemiol e Serviços Saúde 2014;23(1):183-4. doi: 10.5123/S167949742014000100018

11. Durieux N, Pasleau F, Howick J. OCEBM Levels of Evidence Working Group. The Oxford 2011 Levels of Evidence. Group [Internet]. 2011;1(version):5653. [cited 2021 Dec 16]. Available from: https://www.cebm.ox.ac.uk/resources/levels-ofevidence/ocebm-levels-of-evidence

12. Del'Angelo N, Santos Nogueira Góes F, Dalri MCB, Leite AM, Furtado MCC, Scochi CGS. Diagnósticos de enfermagem de prematuro sob cuidados intermediários. Rev Bras Enferm, Brasília 2010 set-out; 63(5): 755-61. doi: 10.1590/S003471672010000500010

13. Santos APS, Silva MLC, Souza NL, Mota GM, França DF. Diagnósticos de enfermagem de recém-nascidos com sepse em uma Unidade de Terapia Intensiva Neonatal. Rev Latinoam Enferm 2014;22(2):255-61. doi: 10.1590/0104-1169.3101.2410

14. Urakawa IT, Kobayashi RM. Identificação do perfil e diagnósticos de enfermagem do neonato com cardiopatia congênita. Revista de Pesquisa Cuidado é Fundamental Online [Internet] 2012 [cited $2021 \mathrm{Dec} 16$ ];4(4):3118-24. Available from: https://www.redalyc.org/pdf/5057/505750895025.pdf

15. Oliveira SIM, Souza NL, Costa e Silva RK. Diagnósticos de enfermagem em recémnascido com alterações glicêmicas. Cogitare Enferm 2013;18(4):702-8. doi: 10.5380/ce.v18i4.34923

16. Herdman TH. Diagnósticos de enfermagem da NANDA: definições e classificação 2015-2017. Porto Alegre: Artmed; 2015. 
17. Gurgel EPP, Rolim KMC, Galvão MTG. Abordagem assistencial ao neonato portador de mielomeningocele segundo o modelo de adaptação de Roy. Rev Esc Enferm USP 2010;44(3):702-7. doi: 10.1590/S0080-62342010000300021

18. Barbosa PMK, Guimarães AA, Santos VC, Anjos KF. Troca de gases prejudicada: intervenções de enfermagem. C\&D - Revista Eletrônica da Fainor 2009;2(1):33-45.

19. Sousa VEC, Montoril MH, Pascoal LM, Lopes MVO. Avaliação da troca gasosa de pacientes com insuficiência cardíaca congestiva. Cogitare Enferm 2010;15(4):681-7. doi: $10.5380 /$ ce.v15i4.20365 